\title{
Torsional Newton-Cartan geometry from Galilean gauge theory
}

\author{
Rabin Banerjee ${ }^{\mathrm{a}, \mathrm{b}}$, Pradip Mukherjee ${ }^{\mathrm{c}, \mathrm{d}}$ \\ ${ }^{a} S$. N. Bose National Centre for Basic Sciences, JD Block, Sector III, Salt \\ Lake City, Kolkata-700 098, India \\ ${ }^{\mathrm{c}}$ Department of Physics, Barasat Government College, \\ 10, KNC Road, Barasat, Kol 700124. \\ ${ }^{\mathrm{b}}$ rabin@bose.res.in \\ ${ }^{\mathrm{d}}$ mukhpradip@gmail .com
}

\begin{abstract}
Using the recently advanced Galilean gauge theory (GGT) we give a comprehensive construction of torsional Newton Cartan geometry. The coupling of a Galilean symmetric model with background NC geometry following GGT is illustrated by a free nonrelativistic scalar field theory. The issue of spatial diffeomorphisn 16, 32 is focussed from a new angle. The expression of the torsionful connection is worked out which is in complete parallel with the relativistic theory. Also smooth transition of the connection to its well known torsionless expression is demonstrated. A complete (implicit) expression of the torsion tensor for the Newton Cartan spacetime is provided where the first order variables occur in a suggestive way. The well known result for the temporal part of torsion is reproduced from our expression.
\end{abstract}

\section{Introduction}

Newton Cartan (NC) geometry [1, 2, 3, 4, 5, 6, 7, 8, 9] is a resurgent area of interest in the literature [10, 11, 12, 13, 14, 15], mainly because of its applications in various crucial topics such as fractional quantum Hall effect (FQHE) [14, 16, 17, 18, 19, 20, 21, 22, 23, Horava - Lifshitz gravity [14, 24, 25], Galilean anomalies and hydrodynamics [26] etc. Inspite of its ubiquity there exists diverse and in some cases conflicting opinions about the details of $\mathrm{NC}$ geometry in the current literature. To cite an instance it will be sufficient to mention the issue of torsion. It appears from [10, 11] that it is not possible 
to obtain torsional NC geometry without including conformal symmetry in the usual gauging of the centrally extended Galilean algebra. However in [24], where similar techniques have been employed, the existence of torsional NC geometry has been reported without the necessity of including conformal symmetry. This ambiguity is not negotiable as torsion is a property of the NC geometry and its existence or otherwise should not depend on whether there is conformal symmetry or not. Another related issue concerns the expression of the torsion in terms of the elements of NC geometry. Many authors give the 'temporal' part only. Full expressions for the torsional connection had been given in [11, 22] but they do not agree. In the latter, torsion is purely temporal and the expression for torsional connection may be criticised from several angles. The central issue is that torsional NC geometry should be viewed as the nonrelstivistic limit of Riemann Cartan spacetime. In most of the existing analysis, this point seems to be obscured.

In fact, the concept of nonrelativistic diffeomorphism invariance (NRDI), which led to the recent understanding of the $\mathrm{NC}$ spacetime, was marred with several problems, of which passage to Galilean symmetry in the flat limit is a representative one. We have shown that this problem disappears if we introduce non relativistic diffeomorphism invariance in a systematic manner. This is done by gauging the global symmetry of the dynamical model in question 1. A general algorithm is developed to couple a Galileo symmetic model with background curvature [13, 14, 29, 32, 34]. This theory, which has been inspired by gauging the Poincare symmetry (Poincare gauge theory) [35, 36, 37, may aptly be called Galilean gauge theory (GGT).

In this paper we will present a thorough analysis of the torsional NC geometry applying GGT. This is a new application where the connection is taken to be general. We will show how metric compatibility can be used to express the general connection in terms of the Dautcourt's symmetric form plus a part which resembles the contortion tensor in Riemann Cartan space. Thus our analysis is able to pass to the known results [13] for symmetric connection. This is a unique feature of the GGT based analysis. We will also express the torsion tensor as a mixed form, where the part containing the first order variables reveal the arbitrariness of the torsion as the effect of the arbitrariness of the spin connection. The temporal part agrees with the usual result [24, 22, 11].

Though our emphasis is on the torsional NC geometry, it will be ad-

\footnotetext{
${ }^{1}$ Note the difference between gauging the symmetry and gauging the algebra 14
} 
vantagous to begin with the coupling of a Galilean invariant free particle theory with general NC manifold. This problem was earlier solved by taking the corresponding relativistic model, then coupling it with Riemann Cartan background and taking the $c \rightarrow \infty$ limit [26]. We find that using GGT it is practically one step job. In the process we will identify the torsional NC elements from GGT. Apart from fixing our notation, this will provide remarkable spin - offs, as we will see.

The lay out of the present paper will now be described. In section 2 we provide an overview of the torsional NC connection, identifying some of the conflicting aspects of the existing results. The next section illustrates the Galilean gauge theory algorithm [29, 32, 14] by taking the nonrelativistic free particle as an example. The fields introduced in the process are connected with general NC geometric structures in this section, following the GGT approach [13]. This enables us to get an action for the free particle coupled with background $\mathrm{NC}$ geometry. This is a new result from Galilean gauge theory, which was derived earlier from a relativistic theory [26] by taking the $c \rightarrow \infty$ limit. Moeover, this gives an independent verification of the corresponding spatially diffeomorphic theory given by GGT [29]. A complete expression of the connection is given in terms of the metric properties and a combination of the torsion tensors, in section 3. This is the central result of the paper which shows the parallel to the Riemann Cartan manifold. Note that the combination of torsion tensors is an analogue of the contorsion tensor in Riemann Cartan geometry . An implicit mixed form of the torsion tensors is obtained where the firsi order variables appear in a suggestive manner. The temporal part of the torsion tensor can easily be isolated. This temporal part reproduces the well-known expression quoted in the literature. A list of the results is provided. The paper ends with concluding remarks in section 5 .

\section{Overview of the problem}

Non-relativistic diffeomorphism invariance (NRDI) has a long history.Cartan first demonstrated that the Newtonian theory of gravity can also be formulated as a geometric theory in a four dimensional manifold which is now called Newton Cartan (NC) spacetime. The NC spacetime is however characterised by two different degenerate metrics. They follow from the $c \rightarrow \infty$ limit of the Riemannian metric. In these theories there is no torsion. In fact existence of torsion along with metric compatibility was argued to be ac- 
companied with non integrability problem [31. When gravity was the focal theme, a symmetric connection proved to be sufficient. But recent analysis requires torsional NC manifold to which field theories symmetric under Galilean transformations are to be coupled.

However, the understanding of torsional connection is not still very clear. To see the point, it will be sufficient to consider a popular form of torsional connection given in 22 .

$$
\Gamma_{\mu \nu}^{\sigma}=\tau^{\sigma} \partial_{\nu} \tau_{\mu}+\frac{1}{2} h^{\sigma \rho}\left(\partial_{\nu} h_{\rho \mu}+\partial_{\mu} h_{\rho \nu}-\partial_{\rho} h_{\mu \nu}\right)
$$

Here $\left\{\tau^{\sigma}, h^{\sigma \rho}, \Gamma_{\mu \nu}^{\sigma}\right\}$ define a torsional NC manifold. Similar connections have been prescribed in [26], [24].

We define the torsion tensor as,

$$
T_{\mu \nu}^{\rho}=\Gamma_{\nu \mu}^{\rho}-\Gamma_{\mu \nu}^{\rho}
$$

This definition is in conformity with our definition of the covariant derivative in the metric form

$$
\nabla_{\mu} A_{\nu}=\partial_{\mu} A_{\nu}-\Gamma_{\nu \mu}^{\rho} A_{\rho}
$$

Using (11), we get

$$
T_{\mu \nu}^{\rho}=\tau^{\rho}\left(\partial_{\mu} \tau_{\nu}-\partial_{\nu} \tau_{\mu}\right)
$$

The torsion tensor is purely temporal. The question arises why torsion should be purely temporal? In this context it may be remembered that [11],starting from gauging the algebra approach, has reported a spatial part of the torsion.

The second observation is the form of the connection. In torsionless geometry, the connection is given by the symmetric Dautcourt form

$$
\Gamma_{\mu \nu}^{D \sigma}=\tau^{\sigma} \partial_{(\mu} \tau_{\nu)}+\frac{1}{2} h^{\sigma \rho}\left(\partial_{\nu} h_{\rho \mu}+\partial_{\mu} h_{\rho \nu}-\partial_{\rho} h_{\mu \nu}\right)
$$

where, a term containing an arbitrary spatial shift is dropped, in order to make the comparision with (1) transparent. The connection(1) can be written as

$$
\Gamma_{\mu \nu}^{\sigma}=\Gamma_{\mu \nu}^{D \sigma}-\frac{1}{2} T_{\mu \nu}^{\sigma}
$$

where $\Gamma_{\mu \nu}^{D \sigma}$ is symmetric and $T^{\sigma}{ }_{\mu \nu}$ is antisymmetric under the exchange of $\mu$ and $\nu$. We find that the symmetric part of the torsional connection is just 
the Dautcourt term, i.e. same as the torsionless connection. One becomes uneasy, for the geodesic motion given by the well known formula

$$
\frac{d^{2} x^{\mu}}{d \lambda^{2}}+\Gamma_{\rho \nu}^{\mu} \frac{d x^{\rho}}{d \lambda} \frac{d x^{\nu}}{d \lambda}=0
$$

indicates that the geometry of the free fall is not affected by torsion. In fact in Riemann Cartan space time with metric compatibility, the connection is given by

$$
\Gamma_{\mu \nu}^{\sigma}=\frac{1}{2} g^{\sigma \rho}\left(\partial_{\nu} g_{\rho \mu}+\partial_{\mu} g_{\rho \nu}-\partial_{\rho} g_{\mu \nu}\right)+C_{\mu \nu}^{\sigma}
$$

where

$$
C_{\mu \nu}^{\sigma}=-\frac{1}{2}\left(T_{\mu \nu}^{\sigma}-T_{\mu}{ }^{\sigma}{ }_{\nu}+T_{\mu \nu}{ }^{\sigma}\right)
$$

is the contorsion tensor. The symmetric part of the connection (8) is given by

$$
\Gamma_{\mu \nu}^{\sigma}=\frac{1}{2} g^{\sigma \rho}\left(\partial_{\nu} g_{\rho \mu}+\partial_{\mu} g_{\rho \nu}-\partial_{\rho} g_{\mu \nu}\right)-\frac{1}{2}\left(T_{\mu \nu}{ }^{\sigma}+T_{\nu \mu}{ }^{\sigma}\right)
$$

We observe that torsion do modify the symmetric part of the connection (8), as shown by (10). The NC manifold with torsion is, in principle obtainable as $c \rightarrow \infty$ limit of the Riemann Cartan manifold. Thus the torsional connection should be of a similar form as (10), where combination of torsion tensor contributes to the symmetric part.

We have discussed some results concerning torsional connection available in the literature [22, 24, 26]. This is, however, sufficient to convince one self that the concept of torsion in NC geometry is plagued with riddles. The reason is not difficult to find. Torsion is best studied by the first order vielbein approach. An appropriate first order formulation is needed; one about which an unanimity is yet to be achieved [11, 12, 29, 24, 28] .

The difficulty in the introduction of torsion in $\mathrm{NC}$ geomtry may be understood by comparing with Riemann-Cartan spacetime which is the torsionful manifold invariant under relativistic diffeomorphism. In Riemann-Cartan spacetime, metric is factorisable in the vielbines. On the contrary, the NC spacetime is characterized by two degenerate metrics. So a direct approach to the vielbein formulation is problematic. One can of course introduce vielbeins and spin connections geometrically but such diffeomorphic spacetime manifold is vaccuous until we define the physics in the tangent space [30]. In 
case of the relativistic manifold both space and time are relative. Physical laws operating locally are the laws of special theory of relativity. Since space and time are both relative, the Riemann-Cartan spacetime can be foliated arbitrarily and local time direction can be defined normal to the spacelike leaves of the foliation. The situation in non relativistic diffeomorphism is very difficult. The absolute concept of Newtonian time is manifested in the fact that there is a unique foliation of the $\mathrm{NC}$ spacetime where Euclidean geometry holds locally in space with absolute time marching on [31] 2. In a given construction, one can fix this foliation for once. The corresponding coordinates are called the adapted coordinates. The properties of this foliation are dictated by the Galilean dynamics. Any arbitrary foliation will not give Galilean symmetry in the locally flat limit and the vielbein formalism will fail to exhibit dual aspects of physical symmetries, namely local galilean symmetry and nonrelativstic diffeomorphism invariance.

Galilean gauge theory approach [29, 13, 32, 14] is just the right one to reach the Galilean frame by its construction. Thus it can tackle the problems of NRDI in a straightforward way. This is no where more apparent than in discussing the torsional NC spacetime, as we will see in the following.

\section{Galilean gauge theory and Newton Cartan spacetime}

Galilean gauge theory as well as its connection with NC geometric elements have reached a more or less well developed stage [29, 13, 32, 14] over the last one and half year. It is thus unnecessary to give a general review of the method. On the other hand, an illustration of the algorithm with the help of a Galilean symmetric model as an example will be appropriate. This will help us to fix the notation and also provide new insights. We choose the example of a free particle moving nonrelativistically. The coupling of such a theory with $\mathrm{NC}$ background will emerge naturally as a consequence of the algorithm. This is a new result in the context of GGT and reproduces earlier results obtained by taking nonrelativisctic limit of a relativistic theory [26].

We consider the Schrodinger field action

$$
S=\int d t \int d^{3} x\left[\frac{i}{2}\left(\psi^{*} \partial_{0} \psi-\psi \partial_{0} \psi^{*}\right)-\frac{1}{2 m} \partial_{k} \psi^{*} \partial_{k} \psi\right] .
$$

\footnotetext{
${ }^{2}$ Globally this spatial slice may be curved
} 
which is invariant under the Galilean transformations,

$$
x^{\mu} \rightarrow x^{\mu}+\xi^{\mu}
$$

where $\xi^{\mu}$ is given by $\xi^{0}=-\epsilon$ and $\xi^{i}=\eta^{i}-v^{i} t$, with $\eta^{i}=\epsilon^{i}+\lambda^{i}{ }_{j} x^{j}$ The constant parameters $\epsilon, \epsilon^{i}, \lambda^{i j}$ and $v^{i}$ respectively represent time and space translation, spatial rotations and galilean boosts. $\lambda^{i j}$ are antisymmetric under interchange of the indices. If these parameters are elevated to functions of space and time, the corresponding symmetry will involve local Galilean transformations.

The first step is to write the corresponding locally Galilean symmetric model in terms of local coordinates. One can readily write this, following the algorithm given in [29, 32, 14], as

$$
S=\int d x^{0} d^{3} x \frac{\operatorname{det}\left(\Sigma_{a}{ }^{k}\right)^{-1}}{\Sigma_{0}{ }^{0}}\left[\frac{i}{2}\left(\psi^{*} \nabla_{0} \psi-\psi \nabla_{0} \psi^{*}\right)-\frac{1}{2 m} \nabla_{a} \psi^{*} \nabla_{a} \psi\right]
$$

The time coordinate in the local system will be denoted by 0 and the space coordinates by $a$. Collectively, the local coordinates will be denoted by the initial letters of the Greek alphabet (i.e. $\alpha, \beta$ etc.). In order to restore the invariance of the theory (13) under local galilean transformations, we have replaced ordinary derivatives by the local covariant derivatives

$$
\begin{aligned}
\nabla_{0} \psi & =\Sigma_{0}{ }^{0} D_{0} \psi+\Sigma_{0}{ }^{k} D_{k} \psi \\
\nabla_{a} \psi & =\Sigma_{a}{ }^{k} D_{k} \psi .
\end{aligned}
$$

where,

$$
\begin{aligned}
D_{k} \psi & =\partial_{k} \psi+i B_{k} \psi \\
D_{0} \psi & =\partial_{t} \psi+i B_{0} \psi
\end{aligned}
$$

are the covariant drivatives in the global coordinates.

The new fields $B_{\mu}$ and $\Sigma_{\alpha}{ }^{\mu}$ introduced above have crucial physical significance.The gauge fields $B_{0}$ and $B_{k}$ correspond to gauging the rotations and galilean boosts. They have the structures,

$$
\begin{aligned}
B_{k} & =\frac{1}{2} B_{k}^{a b} \omega_{a b}+B_{k}^{a 0} \omega_{a} \\
B_{0} & =\frac{1}{2} B_{0}^{a b} \omega_{a b}+B_{0}^{a 0} \omega_{a}
\end{aligned}
$$


where $\omega_{a b}$ and $\omega_{a}$ are respectively the generators of rotations and Galileo boosts. The fields $\Sigma_{\alpha}{ }^{\mu}$, 'rotate' the global covariant derivatives (15) into the local covariant drivatives (14). The required transformations of $\Sigma_{\alpha}{ }^{\mu}$ are given by [14],

$$
\begin{aligned}
& \delta_{0} \Sigma_{0}{ }^{k}=-\xi^{\nu} \partial_{\nu} \Sigma_{0}{ }^{k}+\Sigma_{0}{ }^{\nu} \partial_{\nu} \xi^{k}-v^{b} \Sigma_{b}{ }^{k} \\
& \delta_{0} \Sigma_{a}{ }^{k}=-\xi^{\nu} \partial_{\nu} \Sigma_{a}{ }^{k}+\Sigma_{a}{ }^{\nu} \partial_{\nu} \xi^{k}-\lambda_{a}{ }^{b} \Sigma_{b}{ }^{k}
\end{aligned}
$$

These transformations show that the lower index of $\Sigma_{\alpha}{ }^{\mu}$ transform under the Galilean transformations, whereas the upper index transform under diffeomorphism, $x^{\mu} \rightarrow x^{\mu}+\xi^{\mu}$. This observation allows one to think (13) as a theory in 4-dim curved space time.

It is also possible to define the curvature tensor by adopting the same path followed in Poincare gauge theory. We can define a field tensor from the commutator of the covariant derivatives given in (14). The curvature tensor may be abstracted from this field tensor. Of course nontrivial difficulty may arise due to the peculiarity of the nonrelativistic aspects. This may be pursued as an independent work but in the present paper we are studying geometrical interpretation, for which dynamics of the gauge fields is not necessary.

The geometric interpretation run as follows. The action (13) can be interpreted as a field thory in curved NC background. The local coordinates $x^{\alpha}$ label the tangent space and the global coordinates $x^{\mu}$ chart the background curved spacetime. The $\Sigma$ fields are the vielbein with $\Lambda$ as their inverse. They satisfy

$$
\Sigma_{\alpha}{ }^{\mu} \Lambda_{\mu}{ }^{\beta}=\delta_{\alpha}^{\beta}, \quad \Sigma_{\alpha}{ }^{\mu} \Lambda_{\nu}{ }^{\alpha}=\delta_{\nu}^{\mu}
$$

The spatial part $\Lambda_{k}{ }^{a}$ is the inverse of $\Sigma_{a}{ }^{k}$.

It has been proved that the 4-dim space referred above is NC manifold. We begin with the definitions of the metric [13],

$$
h^{\mu \nu}=\Sigma_{a}{ }^{\mu} \Sigma_{a}{ }^{\nu}
$$

and the one form

$$
\tau_{\mu}=\Lambda_{\mu}{ }^{0}
$$

where $\Lambda_{\mu}{ }^{\alpha}$ is defined in (18). In our coordinate system $\tau^{\mu}=\Sigma_{0}{ }^{0}, \Sigma_{0}{ }^{k}$. Also, since $\Sigma_{a}^{0}=0$,

$$
h^{\mu 0}=\Sigma_{a}{ }^{\mu} \Sigma_{a}{ }^{0}=h^{0 \mu}=0
$$


Using the transformations (17) and equation (18), we can show that both $h^{\mu \nu}$ and $\tau_{\mu}$ satisfy appropriate trnsformations under diffeomorhism of the manifold.

From the above definition we get

$$
h^{\mu \nu} \tau_{\nu}=0
$$

which shows that the metric $h^{\mu \nu}$ is degenerate. We can, nevertheless, define a timelike vector $\tau^{\mu}$ as

$$
\tau^{\mu}=\Sigma_{0}^{\mu} \quad ; \quad \tau_{\mu} \tau^{\mu}=1
$$

and the covariant tensor

$$
h_{\nu \rho}=\Lambda_{\nu}^{a} \Lambda_{\rho}^{a}
$$

. Clearly,

$$
\begin{aligned}
h_{\mu \nu} \tau^{\nu} & =\Lambda_{\mu}{ }^{a} \Lambda_{\nu}{ }^{a} \Sigma_{0}{ }^{\nu} \\
& =\Lambda_{\mu}{ }^{a} \delta_{0}^{a} \\
& =0
\end{aligned}
$$

Finally

$$
h^{\mu \lambda} h_{\lambda \nu}=\delta_{\nu}^{\mu}-\tau^{\mu} \tau_{\nu}
$$

Thus $h^{\mu \nu}, \tau_{\nu}$ define the elements of the NC geometry. It will be useful to understand the meaning of this geometry. For example, one may enquire about the definition of proper distance. In Newtonian mechanics time is absolute and such distance can only be meaningful if an event in NC manifold is spacelike. The element $\tau_{\mu}$ determines whether an event $\xi^{\mu}$ is timelike $\left(\sqrt{\tau_{\mu} \tau_{\nu} \xi^{\mu} \xi^{\nu}}>0\right)$ or spacelike $\left(\sqrt{\tau_{\mu} \tau_{\nu} \xi^{\mu} \xi^{\nu}}=0\right)$. If the event is spacelike, one can define a distance unambiguously. The distance is defined as $\sqrt{h^{\mu \nu} \lambda_{\mu} \lambda_{\nu}}$, where $\xi^{\mu}=h^{\mu \nu} \lambda_{\nu}$ [33]. Interestingly, the Galilean gauge theory naturally arrives at the coordinate system where the direction of time flow matches with the absolute time. Here, events on the foliation have unique distance where time is not involved. This can be verified from the metric elements given in (21).

It is now straightforward to verify that the action (13) is equivalent to

$$
S=\int d x^{0} d^{3} x \operatorname{det} \Lambda_{\mu}{ }^{\nu}\left[\frac{i}{2} \tau^{\mu}\left(\psi^{*} D_{\mu} \psi-\psi D_{\mu} \psi^{*}\right)-\frac{1}{2 m} h^{\mu \nu} D_{\mu} \psi^{*} D_{\nu} \psi\right]
$$


The factor $d x^{0} d^{3} x \operatorname{det} \Lambda_{\mu}{ }^{\alpha}$ is the volume form defined in the NC manifold. The above action is the cherished form of coupling of (11) with NC geometry. This result is in conformity with similar result derived from a relativistic theory by going to the $c \rightarrow \infty$ limit [26].

The action (27) actually leads to yet another interesting finding. Note that the spatial coordinates in our calculation label the spacelike foliation which is unique in the NC spacetime whereas the time coordinate serves as the affine parameter ticking the absolute time. Specialising to our coordinates and putting the time translation to be zero, we recover the action invariant under most general nonrelativistic spatial diffeomorphism: $x^{i} \rightarrow x^{i}+\xi^{i}$. It was earlier shown that $\Sigma_{0}{ }^{0}=$ const in this limit [32, 14] which can be taken to be 1 without any loss of generality. Denoting the determinant of the spatial metric $h_{k l}$ by $h$, we can easily derive from definition (24),

$$
\operatorname{det} \Lambda_{\mu}{ }^{\nu}=\Sigma_{0}{ }^{0} \operatorname{det} \Lambda_{i}^{j}=\sqrt{h}
$$

Now we can write the action (27) as,

$$
\begin{aligned}
S & =\int d x^{0} d^{3} x \sqrt{h}\left[\frac{i}{2}\left(\psi^{*} D_{0} \psi-\psi D_{0} \psi^{*}\right)-\frac{1}{2 m} h^{k l} D_{k} \psi^{*} D_{l} \psi\right. \\
& \left.+\frac{i}{2} \Sigma_{0}{ }^{k}\left(\psi^{*} D_{k} \psi-\psi D_{k} \psi^{*}\right)\right]
\end{aligned}
$$

In deriving the above we have made use of (23) .We find (29) is exactly what GGT predicts [29].

The above action represents the coupling of nonrelativistic matter with $\mathrm{NC}$ background metric. Note that the space time metric $h^{\mu \nu}$ is degenerate. So the usual procedure of defining the energy momentum tensor as a response to metric variation is not applicable here. Indeed it may be recalled that this definition of energy momentum tensor involves $\sqrt{g}$ in the denominator, where $g$ is the determinant of the metric $g_{\mu \nu}$ which vanishes here. Nevertheless, we can construct an energy momentum tensor by using Noether's prescription. This is possible since we follow a field theoretic approach to develop our formalism.

In this connection it may also be mentioned that in the context of study of transport phenomena in Hall fluid, the action (11) was coupled minimally with curved background in [16], supposedly diffeomorphic under arbitrary time dependent spatial transformation. Their theory was (29) without the 
second term 3. Naturally, their theory failed to be invariant under time dependent deformation. Invariance could only be achieved by assuming non canonical transformation for the fields. Also, as we see clearly here, they did not consider the spin connection. The result is, one has to assume a condition between the Galilean boost and the gauge parameter in order to retrive Galilean symmetry in the flat limit. Not only unnatural, such equality reduces symmetry of the system.

\section{Torsional Newton-Cartan geometry}

In the above, the geometric connection (19]- 26) was exploited to develop a first order (vielbein) formalism of the Newton Cartan geometry. Thus via GGT we have been able to construct NC geometry. We have used these metric relations to couple a nonrelativistic free particle theory with the Newton Cartan background. We still left the issue of connection open. In the standard Newton Cartan theory, the connection is symmetric. Earlier, we have shown how to derive the standard NC connection from the Galilean gauge theory by imposing symmetry of the connection as an additional condition [13. But there is nothing in our construction thst demands this symmetry. In this work we will withdraw the assumption of symmetric connecion and only demand metric compatibility. One then hopes that this analysis may naturally lead to the torsional NC geometry and help clarify the concept of torsion in such spaces. The following analysis shows that this hope will not be belied.

We have already derived the metric structure of a general NC theory in terms of the first order theory variables. To proceed further we have to write the affine connection from our theory. The affine connection $\Gamma_{\nu \mu}^{\rho}$ in the first order form is easy to abstract from the vielbein postulate,

$$
\nabla_{\mu} \Lambda_{\nu}^{\alpha}=\partial_{\mu} \Lambda_{\nu}^{\alpha}-\Gamma_{\nu \mu}^{\rho} \Lambda_{\rho}^{\alpha}+B_{\mu \beta}^{\alpha} \Lambda_{\nu}^{\beta}=0
$$

and is given by

$$
\Gamma_{\nu \mu}^{\rho}=\partial_{\mu} \Lambda_{\nu}{ }^{\alpha} \Sigma_{\alpha}{ }^{\rho}+B^{\alpha}{ }_{\mu \beta} \Lambda_{\nu}{ }^{\beta} \Sigma_{\alpha}{ }^{\rho}
$$

where the spin connection $B^{\alpha}{ }_{\mu \beta}$ is introduced in (16). The connection (31) is demonstrated to be metric compatible in [13]. Note in this connection

\footnotetext{
${ }^{3}$ Actually, in [16], there was an external gauge field. But our arguments hold also in the more general case 32 .
} 
that in the torsionless case, the spin connection may be expressed in terms of the vielbeins [10, 13. However in the present torsional example, the spin connections are independent. From this one can expect that torsion may not be expressible in terms of metric variables alone.

In the general case the connection does not have definite symmetry. However we can always split $\Gamma_{\nu \mu}^{\rho}$ in its symmetric (S) and antisymmetric (A) parts,

$$
\Gamma_{\nu \mu}^{\rho}=\Gamma_{\nu \mu}^{S^{\rho}}+\Gamma_{\nu \mu}^{A \rho}
$$

where, $\Gamma_{\nu \mu}^{A^{\rho}}$ and $\Gamma_{\nu \mu}^{S^{\rho}}$ are, respectively, given by

$$
\begin{aligned}
\Gamma_{\nu \mu}^{A^{\rho}} & =\frac{1}{2}\left[\partial_{\mu} \Lambda_{\nu}{ }^{\alpha} \Sigma_{\alpha}{ }^{\rho}-\partial_{\nu} \Lambda_{\mu}{ }^{\alpha} \Sigma_{\alpha}{ }^{\rho}+B^{\alpha}{ }_{\mu \beta} \Lambda_{\nu}{ }^{\beta} \Sigma_{\alpha}{ }^{\rho}-B^{\alpha}{ }_{\nu \beta} \Lambda_{\mu}{ }^{\beta} \Sigma_{\alpha}{ }^{\rho}\right] \\
& =\frac{1}{2}\left[\partial_{\mu} \Lambda_{\nu}{ }^{0} \Sigma_{0}{ }^{\rho}-\partial_{\nu} \Lambda_{\mu}{ }^{0} \Sigma_{0}{ }^{\rho}+\partial_{\mu} \Lambda_{\nu}{ }^{a} \Sigma_{a}{ }^{\rho}-\partial_{\nu} \Lambda_{\mu}{ }^{a} \Sigma_{a}{ }^{\rho}\right. \\
& \left.+B^{a}{ }_{\mu 0} \Lambda_{\nu}{ }^{0} \Sigma_{a}{ }^{\rho}-B^{a}{ }_{\nu 0} \Lambda_{\mu}{ }^{0} \Sigma_{a}{ }^{\rho}+B^{a}{ }_{\mu b} \Lambda_{\nu}{ }^{b} \Sigma_{a}{ }^{\rho}-B^{a}{ }_{\nu b} \Lambda_{\mu}{ }^{b} \Sigma_{a}{ }^{\rho}\right]
\end{aligned}
$$

and

$$
\begin{aligned}
\Gamma_{\nu \mu}^{S \rho} & =\frac{1}{2}\left[\partial_{\mu} \Lambda_{\nu}{ }^{\alpha} \Sigma_{\alpha}{ }^{\rho}+\partial_{\nu} \Lambda_{\mu}{ }^{\alpha} \Sigma_{\alpha}{ }^{\rho}+B^{\alpha}{ }_{\mu \beta} \Lambda_{\nu}{ }^{\beta} \Sigma_{\alpha}{ }^{\rho}+B^{\alpha}{ }_{\nu \beta} \Lambda_{\mu}{ }^{\beta} \Sigma_{\alpha}{ }^{\rho}\right] \\
& =\frac{1}{2}\left[\partial_{\mu} \Lambda_{\nu}{ }^{0} \Sigma_{0}{ }^{\rho}+\partial_{\nu} \Lambda_{\mu}{ }^{0} \Sigma_{0}{ }^{\rho}+\partial_{\mu} \Lambda_{\nu}{ }^{a} \Sigma_{a}{ }^{\rho}+\partial_{\nu} \Lambda_{\mu}{ }^{a} \Sigma_{a}{ }^{\rho}\right. \\
& \left.+B^{a}{ }_{\mu 0} \Lambda_{\nu}{ }^{0} \Sigma_{a}{ }^{\rho}+B^{a}{ }_{\nu 0} \Lambda_{\mu}{ }^{0} \Sigma_{a}{ }^{\rho}+B^{a}{ }_{\mu b} \Lambda_{\nu}{ }^{b} \Sigma_{a}{ }^{\rho}+B^{a}{ }_{\nu b} \Lambda_{\mu}{ }^{b} \Sigma_{a}{ }^{\rho}\right]
\end{aligned}
$$

The form of the symmetric part in terms of the NC structures will now be worked out. Using (20) in (34) we obtain

$$
\begin{aligned}
\Gamma_{\nu \mu}^{S \rho} & =\tau^{\rho} \partial_{(\mu} \tau_{\nu)}+\frac{1}{2}\left[\partial_{\mu} \Lambda_{\nu}{ }^{a} \Sigma_{a}{ }^{\rho}+\partial_{\nu} \Lambda_{\mu}{ }^{a} \Sigma_{a}{ }^{\rho}\right. \\
& \left.+B^{a}{ }_{\mu 0} \Lambda_{\nu}{ }^{0} \Sigma_{a}{ }^{\rho}+B^{a}{ }_{\nu 0} \Lambda_{\mu}{ }^{0} \Sigma_{a}{ }^{\rho}+B^{a}{ }_{\mu b} \Lambda_{\nu}{ }^{b} \Sigma_{\alpha}{ }^{\rho}+B^{a}{ }_{\nu b} \Lambda_{\mu}{ }^{b} \Sigma_{a}{ }^{\rho}\right]
\end{aligned}
$$

To proceed further, note that if we assume the connection to be symmetric, the corresponding theory is torsionless NC theory. This symmetric connection is entirely given by metric quantities [31]. We will see how it is modified 
in presence of torsion. As far as we know, this analysis is a novel one in the literature.

Using (33) and (25) we can write,

$$
\begin{aligned}
h_{\lambda \rho} T_{\mu \nu}^{\rho} & =\left[\partial_{\mu} \Lambda_{\nu}{ }^{a} \Sigma_{a}{ }^{\rho}-\partial_{\nu} \Lambda_{\mu}{ }^{a} \Sigma_{a}{ }^{\rho}\right. \\
& \left.+B^{a}{ }_{\mu 0} \Lambda_{\nu}{ }^{0} \Sigma_{a}{ }^{\rho}-B^{a}{ }_{\nu 0} \Lambda_{\mu}{ }^{0} \Sigma_{a}{ }^{\rho}+B^{a}{ }_{\mu b} \Lambda_{\nu}{ }^{b} \Sigma_{a}{ }^{\rho}-B^{a}{ }_{\nu b} \Lambda_{\mu}{ }^{b} \Sigma_{a}{ }^{\rho}\right] h_{\lambda \rho}
\end{aligned}
$$

where the definition (2) of the torsion has been used. Now, $\Sigma_{a}{ }^{\rho} h_{\lambda \rho}=\Lambda_{\lambda a}$. Equation (36)thus becomes

$$
\begin{aligned}
\partial_{\mu} \Lambda_{\nu}{ }^{a} \Lambda_{\lambda a} & -\partial_{\nu} \Lambda_{\mu}{ }^{a} \Lambda_{\lambda a}+B^{a}{ }_{\mu 0} \Lambda_{\nu}{ }^{0} \Lambda_{\lambda a}-B^{a}{ }_{\nu 0} \Lambda_{\mu}^{0} \Lambda_{\lambda a} \\
& +B^{a}{ }_{\mu b} \Lambda_{\nu}{ }^{b} \Lambda_{\lambda a}-B^{a}{ }_{\nu b} \Lambda_{\mu}{ }^{b} \Lambda_{\lambda a}=h_{\lambda \rho} T_{\mu \nu}^{\rho}
\end{aligned}
$$

Permuting the indices $\mu, \nu, \lambda$ cyclically twice and subtracting the first outcome from the second, we get,

$$
\begin{aligned}
B^{a}{ }_{\mu b} \Lambda_{\nu}{ }^{a} \Lambda_{\lambda b} & +B^{a}{ }_{\nu a} \Lambda_{\mu}{ }^{a} \Lambda_{\lambda b}=-\partial_{\nu} \Lambda_{\lambda a} \Lambda_{\mu}{ }^{a}+\partial_{\lambda} h_{\mu \nu}-\partial_{\mu} \Lambda_{\lambda a} \Lambda_{\nu}{ }^{a} \\
& -\left[\left(B_{\mu}{ }^{a 0} \tau_{\lambda}-B_{\lambda}{ }^{a 0} \tau_{\mu}\right) \Lambda_{\nu a}-\left(B_{\nu}{ }^{a 0} \tau_{\lambda}-B_{\lambda}{ }^{a 0} \tau_{\nu}\right) \Lambda_{\mu a}\right] \\
& +h_{\mu \rho} T_{\nu \lambda}^{\rho}-h_{\nu \rho} T_{\lambda \mu}^{\rho}
\end{aligned}
$$

Substituting (38) in (35) we get the desired expression for the symmetric part of the connection,

$$
\begin{aligned}
\Gamma_{\mu \nu}^{S \sigma} & =\tau^{\sigma} \partial_{(\mu} \tau_{\nu)}+\frac{1}{2} h^{\sigma \rho}\left(\partial_{\nu} h_{\rho \mu}+\partial_{\mu} h_{\rho \nu}-\partial_{\rho} h_{\mu \nu}\right)+h^{\sigma \lambda} K_{\lambda(\mu} \tau_{\nu)} \\
& -\frac{1}{2} h^{\sigma \lambda}\left[h_{\mu \rho} T_{\lambda \nu}^{\rho}+h_{\nu \rho} T_{\lambda \mu}^{\rho}\right]
\end{aligned}
$$

where, the penultimate term consists of an arbitrary shift by the two form $\mathrm{K}$,

$$
K_{\lambda \mu}=\left[B_{\mu}^{a 0} \Lambda_{\lambda a}-B_{\lambda}^{a 0} \Lambda_{\mu a}\right]
$$

Expression (39) is the symmetric part of the connection for the torsional NC spacetime. If we additionaly impose the symmetry

$$
\Gamma_{\mu \nu}^{\rho}=\Gamma_{\nu \mu}^{\rho}
$$


the connection becomes

$$
\Gamma_{\mu \nu}^{D \sigma}=\tau^{\sigma} \partial_{(\mu} \tau_{\nu)}+\frac{1}{2} h^{\sigma \rho}\left(\partial_{\nu} h_{\rho \mu}+\partial_{\mu} h_{\rho \nu}-\partial_{\rho} h_{\mu \nu}\right)+h^{\rho \lambda} K_{\lambda(\mu} \tau_{\nu)}
$$

which is Dautcourt's formula for the connection in standard (torsionless) NC spacetime.

Coming back to the torsional NC geometry we can easily see that the general connection can be split as

$$
\Gamma_{\mu \nu}^{\rho}=\Gamma_{\mu \nu}^{D \sigma}+C_{\mu \nu}^{D \sigma}
$$

where,

$$
C_{\mu \nu}^{D \rho}=-\frac{1}{2}\left[T_{\mu \nu}^{\rho}-h^{\rho \lambda} h_{\mu \sigma} T_{\lambda \nu}^{\sigma}+h^{\rho \lambda} h_{\nu \sigma} T_{\mu \lambda}^{\sigma}\right]
$$

Note that $C^{D \sigma}{ }_{\mu \nu}$ has no definite symmetry in conformity to the fact that connection is of the most general type.

Equation (43) is one of the most significant results of the present report. We see that the symmetric part of the connection (39) contain two parts. The first one is the Dautcourt connection, the appropriate connection for the torsionless case. The second part originates due to torsion. In torsional NC geometry, the symmetric part of the connection is influenced by the torsion. This influence has no temporal part because, quite generally, identity (26) enforces it in the spatial sector. The definition of straight line as a line parallel to itself is thus influenced by the torsion. This is expected because in Riemann Cartan space similar phenomenon exists, the difference lies in the fact that the latter is not confined to spatial sector. The similarity and the difference are both expected. Torsional NC space time is in principle obtainable as $c \rightarrow \infty$ limit of the Riemann Cartan space. The difference is explained by the existence of absolute time in $\mathrm{NC}$ geometry.

Secondly, a scrutinee of the result shows that there is an arbitrary two form in the expression of the symmetric connection, just like the torsionless $\mathrm{NC}$ theory. In fact the torsionless theory should be recovered from the more general theory by putting torsion to be zero. Surprisingly this requirement is not much emphasised in the literature.

Our next task is to express the torsion (2). Primarily, we get from (33) that

$$
\begin{aligned}
T_{\mu \nu}^{\rho} & =\tau^{\rho}\left(\partial_{\mu} \tau_{\nu}-\partial_{\nu} \operatorname{tau}_{\mu}\right)+\Sigma_{a}{ }^{\rho}\left[\partial_{\mu} \Lambda_{\nu}{ }^{a}-\partial_{\nu} \Lambda_{\mu}{ }^{a}\right. \\
& \left.+B^{a}{ }_{\mu 0} \Lambda_{\nu}{ }^{0}-B^{a}{ }_{\nu 0} \Lambda_{\mu}{ }^{0}+B^{a}{ }_{\mu b} \Lambda_{\nu}{ }^{b}-B^{a}{ }_{\nu b} \Lambda_{\mu}{ }^{b}\right]
\end{aligned}
$$


As one can see, the temporal part is

$$
\tau_{\rho} T^{\rho}{ }_{\mu \nu}=\left(\partial_{\mu} \tau_{\nu}-\partial_{\nu} \tau_{\mu}\right)
$$

as a consequence of

$$
\tau_{\rho} \Sigma_{a}{ }^{\rho}=\Lambda_{\rho}{ }^{0} \Sigma_{a}{ }^{\rho}=\delta_{a}^{0}=0
$$

The expression of the temporal part agrees universally.

However (45) also shows that torsion is not purely temporal. The spatial part is given in (45) in the first order variables. Question is, can it be expressed entirely in terms of metric structures. Comparision with Riemann Cartan space once again gives us guideline. It suggests that torsion should be dependent on independent spin connection [38. Below, we will derive an implicit relation involving torsion, where this dependence is manifest.

Starting from the definition (2) we get after a few steps

$$
\begin{aligned}
T^{\rho}{ }_{\mu \nu} & =\tau^{\rho}\left(\partial_{\mu} \tau_{\nu}-\partial_{\nu} \tau_{\mu}\right) \\
& +h^{\rho \sigma} \Lambda_{\sigma a}\left[\left(\partial_{\mu} h_{\nu \lambda}\right) \Sigma_{a}{ }^{\lambda}+h_{\nu \lambda}\left(\partial_{\mu} \Sigma_{a}{ }^{\lambda}\right)-\left(\partial_{\nu} h_{\mu \lambda}\right) \Sigma_{a}{ }^{\lambda}-h_{\mu \lambda}\left(\partial_{\nu} \Sigma_{a}{ }^{\lambda}\right)\right] \\
& +h^{\rho \sigma} \Lambda_{\sigma a}\left[\left(B_{\mu}{ }^{a b} \Lambda_{\nu b}-B_{\nu}{ }^{a b} \Lambda_{\mu b}\right)+\left(B_{\mu}{ }^{a 0} \Lambda_{\nu 0}-B_{\nu}{ }^{a 0} \Lambda_{\mu 0}\right)\right]
\end{aligned}
$$

Now note that

$$
\Sigma_{a}{ }^{\lambda} \Lambda_{\sigma a}=\Sigma_{\alpha}{ }^{\lambda} \Lambda_{\sigma \alpha}-\Sigma_{0}{ }^{\lambda} \Lambda_{\sigma 0}=\delta_{\sigma}^{\lambda}-\tau_{\sigma} \tau^{\lambda} .
$$

When multiplied by $h^{\rho \sigma}$, the result is

$$
h^{\rho \sigma} \Sigma_{a}^{\lambda} \Lambda_{\sigma a}=h^{\rho \lambda}
$$

Contribution from the second term of the right hand side of (49) vanishes. This result will be important in various simplifications.

Now, using (38) we can express

$$
\begin{aligned}
\left(B_{\mu}{ }^{a b} \Lambda_{\nu a} \Lambda_{\sigma b}\right. & \left.-B_{\nu}{ }^{a b} \Lambda_{\mu a} \Lambda_{\sigma b}\right)=\Lambda_{\mu}{ }^{a} \partial_{\sigma} \Lambda_{\nu}{ }^{a}-\Lambda_{\nu}{ }^{a} \partial_{\sigma} \Lambda_{\mu}{ }^{a}+2\left(\partial_{\mu} h_{\sigma \nu}-\partial_{\nu} h_{\sigma \mu}\right. \\
& +\partial_{\nu} \Lambda_{\sigma a} \Lambda_{\mu a}-\partial_{\mu} \Lambda_{\sigma a} \Lambda_{\nu a} \\
& +\left[B_{\mu}{ }^{a 0}\left(\tau_{\sigma} \Lambda_{\nu a}+\tau_{\nu} \Lambda_{\sigma a}\right)-B_{\mu}{ }^{a 0}\left(\tau_{\sigma} \Lambda_{\nu a}+\tau_{\nu} \Lambda_{\sigma a}\right)\right] \\
& +\left(h_{\nu \lambda} T^{\lambda}{ }_{\sigma \mu}-h_{\mu \lambda} T^{\lambda}{ }_{\sigma \nu}+2 h_{\sigma \lambda} T^{\lambda}{ }_{\nu \mu}\right)
\end{aligned}
$$

Substituting (51) and (50) in (48) we obtain after some simplifications,

$$
\begin{aligned}
T^{\rho}{ }_{\mu \nu} & -h^{\rho \sigma} h_{\nu \lambda} T^{\lambda}{ }_{\sigma \mu}+h^{\rho \sigma} h_{\mu \lambda} T^{\lambda}{ }_{\sigma \nu}=\tau^{\rho}\left(\partial_{\mu} \tau_{\nu}-\partial_{\nu} \tau_{\mu}\right)-h^{\rho \sigma}\left(\partial_{\mu} h_{\nu \sigma}-\partial_{\nu} h_{\mu \sigma}\right) \\
& +h^{\rho \sigma}\left[\Lambda_{\nu a} \partial_{\sigma} \Lambda_{\mu a}-\Lambda_{\mu a} \partial_{\sigma} \Lambda_{\nu a}+2 B_{\sigma}{ }^{a b} \Lambda_{\nu a} \Lambda_{\mu b}\right]
\end{aligned}
$$


Note that first order variables occur in a remarkable combination, $\Lambda_{\nu a} \partial_{\sigma} \Lambda_{\mu a}-$ $\Lambda_{\mu a} \partial_{\sigma} \Lambda_{\nu a}+2 B_{\sigma}{ }^{a b} \Lambda_{\nu a} \Lambda_{\mu b}$. We can extend it to

$$
\begin{aligned}
\Lambda_{\nu a} \partial_{\sigma} \Lambda_{\mu a}-\Lambda_{\mu a} \partial_{\sigma} \Lambda_{\nu a}+2 B_{\sigma}{ }^{a b} \Lambda_{\nu a} \Lambda_{\mu b} & +B_{\sigma}{ }^{a 0} \Lambda_{\nu a} \Lambda_{\mu 0}-B_{\sigma}{ }^{a 0} \Lambda_{\mu a} \Lambda_{\nu 0} \\
& -\left[B_{\sigma}{ }^{a 0} \Lambda_{\nu a} \Lambda_{\mu 0}-B_{\sigma}{ }^{a 0} \Lambda_{\mu a} \Lambda_{\nu 0}\right][53)
\end{aligned}
$$

Now

$$
\begin{aligned}
\Lambda_{\nu a} \partial_{\sigma} \Lambda_{\mu a}-\Lambda_{\mu a} \partial_{\sigma} \Lambda_{\nu a}+2 B_{\sigma}{ }^{a b} \Lambda_{\nu a} \Lambda_{\mu b} & +B_{\sigma}{ }^{a 0} \Lambda_{\nu a} \Lambda_{\mu 0}-B_{\sigma}{ }^{a 0} \Lambda_{\mu a} \Lambda_{\nu 0} \\
& =\Lambda_{\nu}{ }^{a} D_{\sigma} \Lambda_{\mu}{ }^{a}-\Lambda_{\mu}{ }^{a} D_{\sigma} \Lambda_{\nu}{ }^{a}
\end{aligned}
$$

where, $D_{\sigma} \Lambda_{\mu}{ }^{a}$ is the covariant derivative with respect to spin connection. Substitution of the above in (52), we get

$$
\begin{aligned}
T_{\mu \nu}^{\rho} & -h^{\rho \sigma} h_{\nu \lambda} T_{\sigma \mu}^{\lambda}+h^{\rho \sigma} h_{\mu \lambda} T_{\sigma \nu}^{\lambda}=\tau^{\rho}\left(\partial_{\mu} \tau_{\nu}-\partial_{\nu} \tau_{\mu}\right)-h^{\rho \sigma}\left(\partial_{\mu} h_{\nu \sigma}-\partial_{\nu} h_{\mu \sigma}\right) \\
& +h^{\rho \sigma}\left(\Lambda_{\nu}{ }^{a} D_{\sigma} \Lambda_{\mu}{ }^{a}-\Lambda_{\mu}{ }^{a} D_{\sigma} \Lambda_{\nu}{ }^{a}\right)-h^{\rho \sigma}\left[\left(B_{\sigma}{ }^{a 0} \Lambda_{\nu a} \Lambda_{\mu 0}-B_{\sigma}{ }^{a 0} \Lambda_{\mu a} \Lambda_{\nu 0}{ }^{5} 55\right)\right.
\end{aligned}
$$

In the following let us summarise the findings of the present paper:

1. The coupling of a nonrelativistic matter theory with the general NC background has been achievd by GGT.

2. As a special case of the above we have studied the corresponding spatially diffeomorphic action. We found that the prescription of GGT is favoured, rather than that of [16].

3. A full expression of the torsional connection is obtained as a sum of the Dautcourt form and a combination of the torsion which can be compared with the contorsion tensor in Riemann - Cartan spacetime. The symmetric part of it is modified by the contribution coming from this combination.

4. It should be stressed that we followed the standard method of gauging for obtaining the torsion. In particular we have assigned compensating fields for time and space translation as well as rotations and boosts (see equation (16)). The nonrelativistic spin connection defined there is used to construct the connection (31). The torsion is just the antisymmetric part of this connection. The Dautcourt connection does not play any role in this derivation. The metric forms including the Dautcourt connection and the contorsion have been invoked to trace a parallel between Riemann Cartan spacetime and the torsional NC spacetime. 
5. The 'temporal' part of the torsion can be identified,

$$
\tau_{\rho} T_{\mu \nu}^{\rho}=\left(\partial_{\mu} \tau_{\nu}-\partial_{\nu} \tau_{\mu}\right)
$$

This expression is well known in the literature [11, 22, 24].

6. Our formulation is based on a systematic analysis of NC geometry. This is noteworthy, for sometimes the torsional connection has been assumed as an ansatz [22] where torsion is purely temporal. The theoretical premises of the ansatz are not quite obvious. Often the deductions are quite intricate and there is no clear algorithm to understand the connections between the vielbein and metric formulations of the NC geometry [11]. On the contrary, the Galilean gauge theory offers a complete and natural formulation of the torsional Newton Cartan geometry, as has been shown here.

\section{$5 \quad$ Concluding remarks}

In the above analysis we have demonstrated how torsional Newton Cartan (NC)geometry can be obtained in Galilean gauge theory (GGT), a recent method of obtaining non relativistic diffeomorphism, formulated in [29] and applied successfully to a host of phenomena [13, 14, 32, 34]. There are several approaches to the problem. However, there are differences of opinion in current literature [11, 22, 24] about various aspects of the first order formalism of NC geometry. We have, therefore, derived the general metric structure from an example. This served to fix our notations. But remarkably, we get new results in the process. We started from a nonrelativistic free particle theory having Galilean symmetry in thr flat space. The algorithm of GGT was applied to eventually couple it with $\mathrm{NC}$ background and one will hardly

miss the relative ease with which the coupling was done. The structure of the coupled theory matched exactly with an earlier result [26], obtained by the approach of taking the $c \rightarrow \infty$ limit of a relativistic model.

Anothr spin off is a resolution of the problem of exact nonrelativistic diffeomorphism invariant action for a particle. The problem is well known. From GGT we have earlier derived an action [29, 32, 14] which had the correct symmetry dictated by appropriate transformations of the fieds under nonrelativistic diffeomorphism. The result from GGT differs from that of [16], where however some of the fields have to transform anomalously for the invariance 
to remain valid. The model proposed in [16] also did not have a proper flat limt -the gauge parameter gets mingled up with the boost parameter. This not only reduces symmetry but also makes the curved space coupling of a free theory impossible. In this paper we find that a proper coupling with a background curvature in space which follows from the corresponding theory in NC space time agrees with the form provided by GGT.

As we have considered genral NC geometry, we found the general expression of the conection in terms of the metric variables and torsion. It has a clear division in terms of the well known Dautcourt form and a combination of the torsion tensor which formally resembles with the contortion tensor in Riemann Cartan space. From this expression (43) the passage to torsionless limit is crystal clear.

We also obtained expression for torsion. The temporal part of the torsion tensor agrees with the well known result in the literature. But our analysis clearly shows that it is not the whole story, There is a spatial part of the torsion tensor which is not entirely expressible in terms of the metric variables. This is also similar to relativistic theories. The metric variables were extracted as far as possible and an implicit relation for torsion 55 could be provided which explains our inability. The point is that in a general torsional manifold the turning of the orthogonal local frame contributes to the torsion.

The construction of $\mathrm{NC}$ geometry with torsion is provided by using Galilean gauge theory. In a subject riddled with so many confusing points, our results stand in their own merit.As GGT is based on localising the symmetry of a dynamical theory, the essence of the Galilieo Newton concept of spacetime is inbuilt in it. Once again, we observe the merit of it in studying nonrelativistic diffeomorphism.

\section{References}

[1] E. Cartan, Sur les variétés à connexion affine et la théorie de la relativité généralisée. (première partie), Annales Sci.Ecole Norm.Sup. 40 (1923) 325-412.

[2] E. Cartan, Sur les variétés à connexion affine et la théorie de la relativité généralisée. (première partie) (Suite)., Annales Sci.Ecole Norm.Sup. 41 (1924) 1-25.

[3] P. Havas, Rev. Mod. Phys. 36,(1964),938. 
[4] J. L. Anderson: Principles of Relativity Physics, Academic Press, London 1967.

[5] A. Trautman, "Theories of Space, Time and Gravitation" in Lectures on General Relativity, S. Deser and K.W. Ford, eds., Prentice-hall, Englewood Cliffs, 1965.

[6] K. Kuchař, Phys. Rev. ,22D,6,(1980),1285.

[7] G. Dautcourt: "Die Newtonske Gravitationstheorie als Strenger Grenzfall der Allgemeinen Relativitätheorie", Acta Phys. Pol. $\mathbf{2 5 , 5 , ( 1 9 6 4 ) , 6 3 7 .}$

[8] J. Ehlers: "On Limit Relations between, and Approximative Explanations of, Physical Theories", in Logic, Methodology and Philosophy of Science, VII, B. Marcus et al., eds., Elsiever, Amsterdam (1986),405.

[9] D.B. Malament: "Newtonian Gravity, Limits, and the Geometry of Space", in From Quarks to Quasars, ed. R.G. Colodny, Pittsburgh University Press, Pittsburgh (1986)p.181.

[10] R. Andringa , E. Bergshoeff, S. Panda and Mees de Roo, Class. Quantum Grav. 28105011 (2011).

[11] E. A. Bergshoeff, J. Hartong, J. Rosseel, Class. Quantum Grav. 28 105011 (2011).

[12] D. T. Son, "Newton-Cartan Geometry and the Quantum Hall Effect", arXiv:1306.0638.

[13] R. Banerjee, A. Mitra, P. Mukherjee, Class. Quantum Grav. 32, 045010 (2015).

[14] R. Banerjee, P. Mukherjee, Phys. Rev. D93, 085020 (2016).

[15] X. Bekaert, K. Morand , Journal of Mathematical Physics 57, 022507 (2016)

[16] D.T. Son and M. Wingate, Annals. of. Physics. 321, 197-224 (2006).

[17] G. Y. Cho, Y. You and E. Fradkin Phys. Rev. B90 (2014) 115139. 
[18] C. Hoyos and D. T. Son, Phys.Rev.Lett. 108 (2012) 066805.

[19] S. Moroz and C. Hoyos, Phys. Rev. B 91, 064508 (2015).

[20] S. Moroz, C. Hoyos, L, Radzihovsky,Phys. Rev. B 91, 195409 (2015).

[21] T. Can, M. Laskin, and P. Wiegmann, Phys.Rev.Lett. 113 (2014) 046803.

[22] M. Geracie, D. T. Son, Chaolun Wu and Shao-Feng Wu, Phys. Rev. D91, 045030 (2015).

[23] Chaolun Wu and Shao-Feng Wu, JHEP 1501 (2015) 120

[24] J. Hartong, N. A. Obers, JHEP 1507 (2015) 155

[25] Oleg Andreev, Michael Haack, and Stefan Hofmann, Phys. Rev. D 89, 064012 (2014).

[26] K. Jensen and A. Karch, JHEP 04 (2015) 155.

[27] Tomas Brauner, Solomon Endlich, Alexander Monin, Riccardo Penco , Phys.Rev. D90 (2014) no.10, 105016

[28] M Geracie, K. Prabhu, M. M. Roberts J. Math. Phys. 56, 103505 (2015)

[29] R. Banerjee, A. Mitra, P. Mukherjee, Phys. Lett. B 737, 369-373 (2014).

[30] S. Weinberg, "Gravitation and cosmology: principles and applications of the general theory of relativity. Vol. 1. New York: Wiley, 1972.".

[31] H.P. Künzle, Ann. Inst. Henry Poincaré, 42,(1972),337.

[32] R. Banerjee, A. Mitra, P. Mukherjee, Phys. Rev. D 91, 084021 (2015).

[33] J. Christian, Phys. Rev. D56, 4844 (1997).

[34] A. Mitra, "Weyl rescaled Newton-Cartan geometry from the localization of Galilean and scale symmetries", arXiv:1508.03207 (2015).

[35] R. Utiyama, Phys. Rev. 101 (1956) 1597.

[36] T. W. B. Kibble, J. Math. Phys. 2 (1961) 212. 
[37] D. W. Sciama "On the analogy between charge and spin in general relativity, in: Recent Developments in General Relativity, Festschrift for Infeld (Pergamon Press, Oxford; PWN, Warsaw" (1962) pp. 415

[38] Sean Carroll, Spacetime and Geometry: An Introduction to General Relativity, Pearson international edition, 2016. 